\title{
Preparation of Molecularly Imprinted Polymers Functionalized with Core-shell Magnetic Nanoparticles for the Recognition of Glycoprotein
}

\author{
He-Ye WANG ${ }^{a}$, Li LI' ${ }^{b}$, Yong-Fu ZHAO ${ }^{c, *}$ \\ Institute of Facilities and Equipment in Agriculture, Jiangsu Academy of Agricultural Sciences, \\ Nanjing, Jiangsu 210014, P. R. China \\ ahywang790907@163.com, ㄹmuzishuiqudou@163.com, 'zyfzyf2002@163.com \\ ${ }^{*}$ Corresponding author
}

Keywords: Molecularly Imprinted Polymers, Core-shell Magnetic Nanoparticles, Glycoprotein.

\begin{abstract}
A method to prepare molecularly imprinted polymers (MIPs) coatings on magnetic $\mathrm{Fe}_{3} \mathrm{O}_{4}$ nanoparticles (MNPs) with core-shell structure for the recognition of glycoprotein was developed. The factors that influence the $\mathrm{Fe}_{3} \mathrm{O}_{4} @$ MIPs, such as polymerization time, extraction time were investigated.
\end{abstract}

\section{Introduction}

Molecularly imprinted polymers (MIPs) are artificial, template-made receptors with the ability to recognize and to specially bind the target molecule [1-2]. The stability, ease of preparation and low cost of these materials have led to their assessment as substitutes for antibodies or enzymes in chemical sensors, catalysis and separations.

Glycoproteins, which occupy more than $50 \%$ of the total proteins in mammalian systems, play key roles in many biological processes, such as molecular recognition, inter- and intra-cellular signaling, and immune response. Besides, many glycoproteins are disease biomarkers and therapeutic targets. Therefore, the imprinting of glycoproteins is of great importance and in high demand.

Boronic acids can covalently interact with cis-diol-containing molecules such as sugars or glycoproteins to form stable cyclic esters in an alkaline aqueous solution while the boronate esters dissociate when the environmental $\mathrm{pH}$ is switched to acidic [3]. This reversible binding has made boronic acids excellent affinity ligands for creating functionalized materials [4-6].

In this study, horseradish peroxidase (HRP) was selected as a glycoprotein model. Boronic acid-functionalized magnetic $\mathrm{Fe}_{3} \mathrm{O}_{4}$ nanoparticles (MNPs) were synthesized for immobilized templates, and then $\mathrm{Fe}_{3} \mathrm{O}_{4} @ \mathrm{MIPs}$ MNPs were prepared.

\section{Materials and Methods}

\section{Materials and Reagents}

Tetraethoxysilane (TEOS) and 3-aminopropyltriethoxylsilane (APTES) were purchased from Alfa Aesar Chemical Company (Tianjin, China). Horseradish peroxidase (HRP), acrylamide(AM), N,N-methylene-bis-(acrylamide) (Bis), ammonium persulfate (APS) N,N,N',N'-tetramethylethylenediamine (TEMED) and 3-aminophenylboronic acid (APBA) were obtained from Sigma-Aldrich (St. Louis, MO, USA). Ferric trichloride hexahydrate, ethylene glycol, anhydrous sodium acetate 1, 6-hexanediamine and glutaraldehyde (GA) were from Sinopharm Chemical Reagent (Shanghai, China). All reagents used were of analytical grade or higher. All commercially available reagents were used without further purification. Water used in all the experiments was purified by a Milli-Q Advantage A10 ultrapure water purification system (Millipore, Milford, MA, USA).

Preparation of the Core-shell Structure $\mathrm{Fe}_{3} \mathrm{O}_{4} @ M I P s$ and Non-imprinting Polymer (NIPs) MNPs

The synthesis procedure of $\mathrm{Fe}_{3} \mathrm{O}_{4} @ \mathrm{SiO}_{2} @ \mathrm{MIPs} \mathrm{MNPs}$ was as shown in Fig. 1.

(1) Synthesis of amino functionalized MNPs. Amino functionalized MNPs were first synthesized 
by the solvent-thermal method [7] with minor modification. Briefly, $2.0 \mathrm{~g}$ ferric trichloride hexahydrate, $13.0 \mathrm{~g} \mathrm{1,6-hexanediamine} \mathrm{and} 4.0 \mathrm{~g}$ anhydrous sodium acetate were mixed with 60 mL glycol in a PTFE-lined autoclave and reacted at $198{ }^{\circ} \mathrm{C}$ for $6 \mathrm{~h}$. The resulting MNPs were rinsed with water and ethanol for 3 times each and then dried at $50{ }^{\circ} \mathrm{C}$.

(2) Preparation of silica shell. To cover the amino functionalized MNPs with a silica shell, 2.7 $\mathrm{mL}$ TEOS and $15.0 \mathrm{~mL}$ ammonium hydroxide were added in $400 \mathrm{~mL}$ ethanol and then the mixture was left to react at room temperature for $20 \mathrm{~min}\left(20{ }^{\circ} \mathrm{C}\right)$. Then $250 \mathrm{mg}$ MNPs were added and the reaction was left for another 20 min. The $\mathrm{Fe}_{3} \mathrm{O}_{4} @ \mathrm{SiO}_{2} \mathrm{MNPs}$ were collected by a magnet at the wall and washed 3 times with ethanol and dried at $50{ }^{\circ} \mathrm{C}$.

(3) Functionalized with boronic acids. 250 mg $\mathrm{Fe}_{3} \mathrm{O}_{4} @ \mathrm{SiO}_{2} \mathrm{MNPs}$ were dispersed in $40 \mathrm{~mL}$ anhydrous toluene containing $2 \mathrm{~mL}$ APTES, the mixture was mechanically stirred (400 rpm) for 12 $\mathrm{h}$ at $120{ }^{\circ} \mathrm{C}$. The resultant APTES-functionalized MNPs washed 3 times with ethanol and dried at $50 \circ \mathrm{C}$. $200 \mathrm{mg}$ APTES-functionalized MNPs was dispersed in $40 \mathrm{~mL}$ 5\% glutaraldehyde in $100 \mathrm{mM}$ sodium phosphate buffer ( $\mathrm{pH}$ 7.0) and the mixture was mechanically stirred for $2 \mathrm{~h}$. The glutaraldehyde-activated MNPs were cleaned by $100 \mathrm{mM}$ sodium phosphate buffer three times then dispersed in $40 \mathrm{~mL} 5 \mathrm{mg} / \mathrm{mL}$ 3-aminophenylboronic acid $100 \mathrm{mM}$ sodium phosphate buffer which contained $1 \%(\mathrm{w} / \mathrm{w})$ sodium cyanoborohydride. After $2 \mathrm{~h}$ reaction, the resultant MNPs were magnetically collected and washed with water and alcohol, dried at $50{ }^{\circ} \mathrm{C}$, and stored for further use.
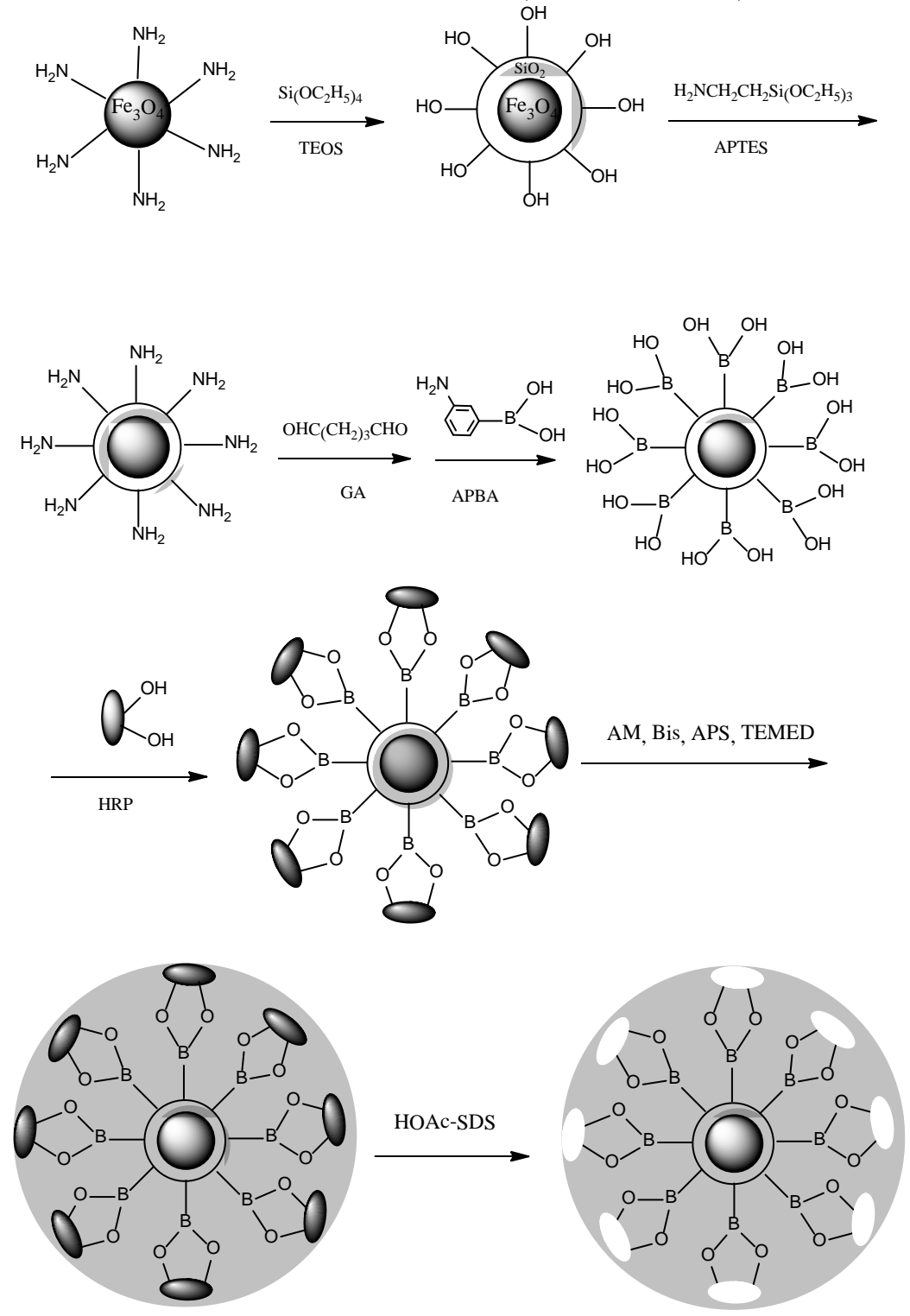

Fig.1 Schematic of preparation of molecularly imprinted polymers functionalized with core-shell magnetic nanoparticles for glycoprotein 
(4) Preparation of the core-shell structure $\mathrm{Fe}_{3} \mathrm{O}_{4} @$ @IPs and $\mathrm{Fe}_{3} \mathrm{O}_{4} @$ NIPs MNPs. HRP was chosen as templates and boronic acid-functionalized MNPs as cores. The functional monomer, cross-linking agent, initiator and accelerator used in this study are acrylamide (AM), N,N-methylene-bis-(acrylamide) (Bis), ammonium persulfate (APS) and N,N,N',N'-tetramethylethylenediamine (TEMED), respectively. 10\% HOAc-10\% sodium dodecyl sulfate (SDS) was then added to remove the template proteins.

Non-imprinted $\mathrm{Fe}_{3} \mathrm{O}_{4} @ N I P s$ MNPs was prepared following the same procedure in the absence of the template protein.

\section{Result and Discussion}

\section{Characterization of $\mathrm{Fe}_{3} \mathrm{O}_{4} @ M I P s$ MNPs}

The size and morphology of the $\mathrm{Fe}_{3} \mathrm{O}_{4} @$ MIPs MNPs were characterized by TEM. As shown in Fig.2, TEM images suggest that the MNPs were well shaped with a diameter of about $100 \mathrm{~nm}$. Because TEM fails to recognize the $\mathrm{Fe}_{3} \mathrm{O}_{4}$ core, the $\mathrm{SiO}_{2}$ shell and the MIPs layer, the thickness of the $\mathrm{SiO}_{2}$ shell and the MIPs coating is unknown.

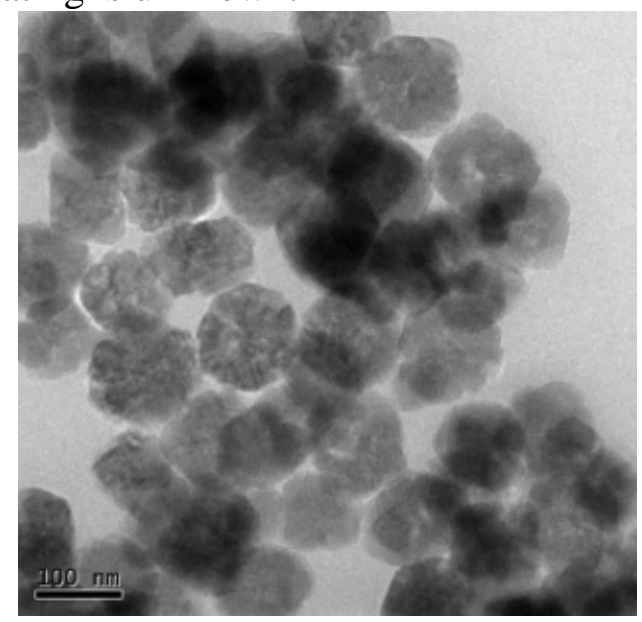

Fig.2 TEM images for $\mathrm{Fe}_{3} \mathrm{O}_{4} @ M I P s$ MNPs

\section{Effect of the Polymerization Time}

Polymerization time is an important factor that determines the $\mathrm{Fe}_{3} \mathrm{O}_{4} @ \mathrm{MIPs}$ MNPs. In this study, four time points, including 12, 15, 18 and $21 \mathrm{~h}$, were compared under otherwise identical conditions. As shown in Fig.3, $15 \mathrm{~h}$ provided the most intense signal for HRP extraction. HRP extraction amount by $\mathrm{Fe}_{3} \mathrm{O}_{4} @$ MIPs MNPs is greater than that of $\mathrm{Fe}_{3} \mathrm{O}_{4} @ N I P s$ MNPs. HRP extracted was detected with chemiluminescence method according to a literature [8].

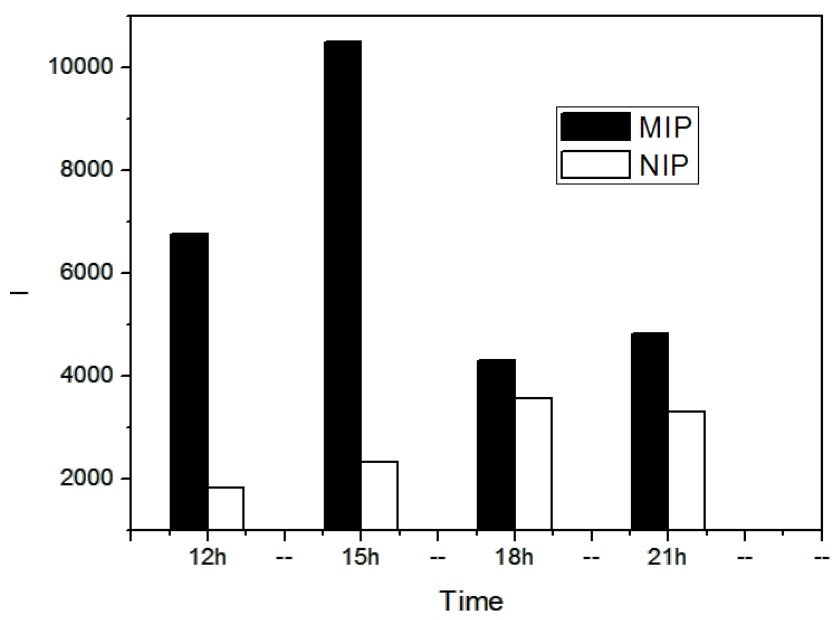

Fig.3 Effect of the polymerization time 


\section{Effect of the Extraction Time}

Four different extraction time points with $\mathrm{Fe}_{3} \mathrm{O}_{4} @$ MIPs MNPs and $\mathrm{Fe}_{3} \mathrm{O}_{4} @ N I P s$ MNPs were studied. As shown in Fig.4, 2.5 h provided the most intense signal, which suggests that longer extraction time favors the extraction efficiency.

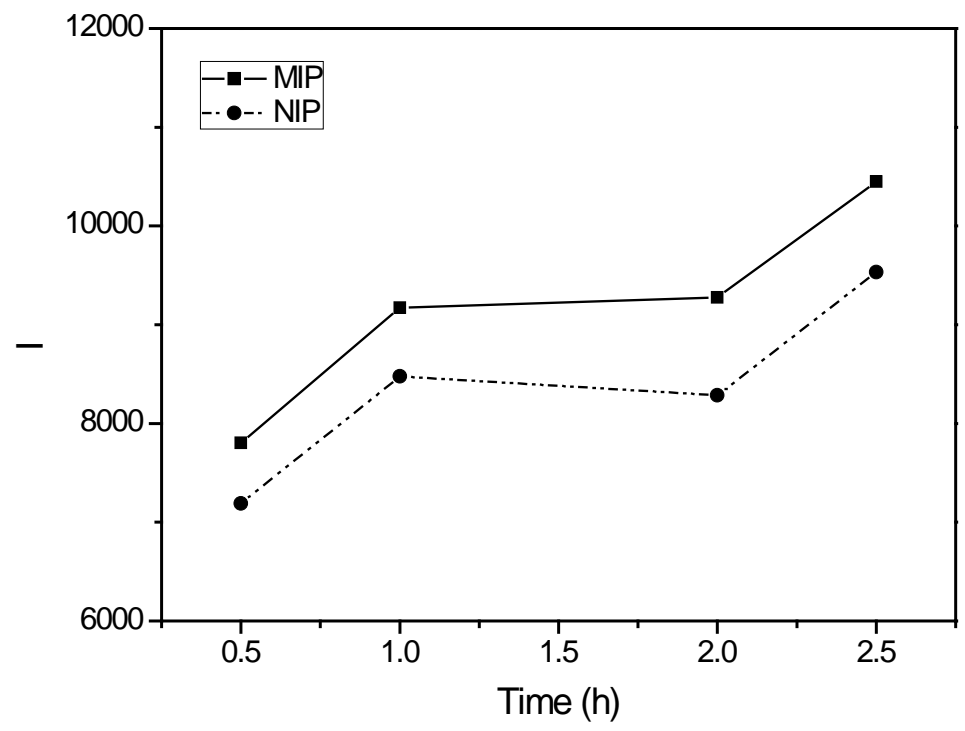

Fig.4 Effect of the extraction time

\section{Conclusion}

In this study, by combining boronate affinity interaction and molecular imprinting, a method was developed to prepare MIP coatings on magnetic $\mathrm{Fe}_{3} \mathrm{O}_{4}$ MNPs with core-shell structure for the recognition of glycoprotein. The factors that influence the $\mathrm{Fe}_{3} \mathrm{O}_{4} @ \mathrm{MIPs}$, such as polymerization time, extraction time were studied.

\section{Acknowledgements}

This research was financially supported by Special Fund for Agro-scientific Research in the Public Interest (201103007).

\section{Reference}

[1]K. Haupt, K. Mosbach, Molecularly imprinted polymers and their use in biomimetic sensors, Chem. Rev. 100 (2000) 2495-2504.

[2]L. Ye, K. Mosbach, Molecular imprinting: synthetic materials as substitutes for biological antibodies and receptors, Chem. Mater. 20 (2008) 859-868.

[3]T.D. James, K.R.A.S. Sandanayake, S. Shinkai, Saccharide sensing with molecular receptors based on boronic acid, Angew. Chem. Int. Ed. Engl. 35 (1996) 1910-1922.

[4]L.B. Ren, Z. Liu, Y.C. Liu, P. Dou, H.Y. Chen, Ring-opening polymerization with synergistic co-monomers: access to a boronate-functionalized polymeric monolith for the specific capture of cis-diol-containing biomolecules under neutral conditions, Angew. Chem. Int. Ed. 48 (2009) 6704-6707.

[5]H.Y. Li, H.Y. Wang, Y.C. Liu, Z. Liu, benzoboroxole-functionalized monolithic column for the selective enrichment and separation of cis-diol containing biomolecules, Chem. Commun. 48 (2012) 4115-4117. 
[6]H.Y. Wang, Z.J. Bie, C.C. Lü, Z. Liu, Magnetic nanoparticles with dendrimer-assisted boronate avidity for the selective enrichment of trace glycoproteins, Chem. Sci. 4 (2013) 4298-4303.

[7]L. Y. Wang, J. Bao, L.Wang, F. Zhang, Y. D. Li, One-potsynthesis and bioapplication of amine-functionalized magnetite nanoparticlesand hollow nanospheres, Chem. Eur. J. 12 (2006) 6341-6347.

[8]L. Li, Y. Lu, Z.J. Bie, H.Y. Chen, Z. Liu, Photolithographic boronate affinity molecular imprinting: a general and facile approach for glycoprotein imprinting, Angew. Chem. Int. Ed. 52 (2013) 7451-7454. 